\title{
Diode Laser Has an Additional Benefit When Used with Conventional Scaling and Root Planning for Chronic Periodontitis Treatment - Comparative Analysis
}

\author{
Himanshu Deswal ${ }^{1}$, Amit Bhardwaj ${ }^{2}$, Harpreet Singh Grover ${ }^{3}$ \\ ${ }^{1}$ ECHS Polyclinic, Ropar, Punjab. India, ${ }^{2,3}$ Department of Periodontology, \\ SGT University, Gurgaon, Haryana, India.
}

\section{ABSTRACT}

\section{BACKGROUND}

Almost $47 \%$ of the population over the age group of 30 is affected by chronic periodontitis. Although the first and gold standard therapy in periodontal treatment is scaling and root planing (SRP), which is a non-surgical approach towards treatment, yet another therapy for the treatment of chronic periodontitis is to irradiate the periodontal pockets with laser. The purpose of this study was to confirm as to whether the use of diode laser $(800-980 \mathrm{~nm})$ as adjunct to scaling and root planning (SRP) improved the results of conventional mechanotherapy in the treatment of chronic periodontitis patients.

\section{METHODS}

In this study we designed our groups in such a way that 40 patients $(20$ males and 20 females) with two deepest nonadjacent pockets $\geq 5 \mathrm{~mm}$ in two different quadrants were selected. In this study each treatment group belonged to a separate quadrant of the mouth. One group was allotted to SRP group while the second one was allotted to SRP + Diode Laser group. Clinical parameters like (Probing Pocket Depth (PPD), Clinical Attachment Level (CAL), Bleeding on Probing (BOP) in chronic periodontitis patients were measured and evaluated at baseline, after 1 month and after 3 months of the treatment. Statistical analysis was also done intra group and inter group.

\section{RESULTS}

From this we infer that PPD was significantly reduced in SRP and SRP + Diode laser groups from baseline and post 1 month $(\mathrm{P}<0.001)$, between baseline and post 3 months $(\mathrm{P}<0.001)$ and between 1 month and 3 months $(\mathrm{P}<0.001)$. There was also significant improvement in CAL in SRP and SRP + Diode Laser groups from baseline and post 1 month $(\mathrm{P}<0.0001)$, between baseline and post 3 months $(\mathrm{P}<0.0001)$ and between 1 month and 3 months $(\mathrm{P}<0.0001)$. BOP also reduced in SRP and SRP + Diode Laser group from baseline. When SRP and SRP + Diode laser groups were compared they showed non statistically significant results but individually both the groups showed statistically significant results.

\section{CONCLUSIONS}

The results of the present study indicate that, comparison of SRP alone group which is a conventional method with SRP as adjunct to Diode laser group i.e., a non-surgical approach showed improvement of CAL and also reduction in PPD and BOP for the treatment of chronic periodontitis patients.

\section{KEY WORDS}

Periodontitis, Laser, Scaling and Root Planing, Adjunct Therapy, Diode Laser
Corresponding Author: Dr. Himanshu Deswal, Dental Officer, ECHS Polyclinic, Ropar Punjab, India. E-mail: deswal706@gmail.com

DOI: $10.14260 / \mathrm{jemds} / 2021 / 523$

How to Cite This Article:

Deswal H, Bhardwaj A, Grover HS. Diode laser has an additional benefit when used with conventional scaling and root planning for chronic periodontitis treatment - comparative analysis. J Evolution Med Dent Sci 2021;10(32):25482553, DOI: 10.14260/jemds/2021/523

Submission 11-01-2021,

Peer Review 07-06-2021,

Acceptance 12-06-2021,

Published 09-08-2021.

Copyright (c) 2021 Himanshu Deswal et al. This is an open access article distributed under Creative Commons Attribution License [Attribution 4.0 International (CC BY 4.0)] 


\section{BACKGROUND}

Periodontitis is a chronic inflammatory disease of infectious origin that leads to destruction of the supporting structures of the teeth, including the periodontal ligament and alveolar bone and in the severe cases, may lead to tooth loss. ${ }^{1}$ Bacterial plaque is the principal etiologic agent in the periodontal disease. Calculus, has rough surface which is capable of retaining the plaque, is permeable to products of the plaque and is present beneath the surface of the plaque. For the successful treatment outcome of disease, subgingival calculus removal is cardinal. ${ }^{2}$ For the management of the periodontal diseases, non- surgical therapy is of utmost importance which comprise of oral hygiene and SRP. Instead of other therapeutical approaches, SRP has been considered as a "gold" standard therapy. ${ }^{3}$ In chronic periodontitis (CP) patients there is significant improvement in the clinical parameters with SRP however, it can be difficult to completely eliminate bacterial deposits. ${ }^{4}$ The areas which are beyond the reach of periodontal instruments like furcation areas, root depressions and deep pockets and also pathogenic bacterial niches in the soft tissue may fail to eliminate completely by the conventional mechanical therapy alone. ${ }^{5}$ Microbial floral composition may be moderately shift with the help of SRP in the treatment of CP, periodontopathic bacteria can persist after SRP particularly in deep periodontal pockets. Recolonization of bacteria of the treated sites can occur in these types of situations. ${ }^{6}$

There is development of many adjunctives to overthrow the restriction of the conventional SRP.7 Among these, laser has been introduced for its detoxification and bactericidal effects and also for its ability to reach the sites where conventional mechanical instruments cannot. ${ }^{8}$ Absorption, reflection, transmission and scattering of laser light are the four ways of how laser light can interact with the target tissue. Light energy gets absorbed and is converted to heat energy which leads to incision, excision or warming and coagulation of the tissue. Although the wavelength of the laser is the primary determining factor of how much energy is imbibed by the tissue, optical properties of the tissue, such as water content, mineral content, and pigmentation, can also affect the extent of energy imbibed. Furthermore, lasers are able to deliver their energy by two wave forms, pulsed and continuous. Continuous wave form lasers carried out huge amounts of energy in an undisturbed, unfluctuating stream potentially emerging in increased heat production. While pulsed wave-form lasers deliver modest amount of thermal energy for short span, so that targeted tissue gets time to cool before the next pulse. Due to the myriad of factors involved with lasers and specific tissue responses, numerous lasers have been developed. ${ }^{9}$

For the treatment of hard tissue in the dentistry, Erbium doped: Yttrium - Aluminium - Garnet (Er: YAG) laser was accepted in 1997. Now a days, several laser systems are present for dental use. For the treatment of soft tissue in the oral cavity several lasers like carbon dioxide $\left(\mathrm{CO}_{2}\right)$, semiconductor diode laser and Neodymium - doped: Yttrium Aluminium - Garnet (Nd: YAG) have been accepted by the US FDA. ${ }^{10}$ Studies have shown that diode laser (DL) can hasten wound healing via the facilitation of promotion of angiogenesis, enhancement of growth factor release, and collagen synthesis at a wavelength between 655 and $980 \mathrm{~nm}$. Additionally, diode laser demonstrates its in-vitro detoxification and bactericidal effects and also hamper ablation of the root surface, which impairs the risk of normal root tissue removal. ${ }^{7}$

All patients were assessed at baseline, after 1 month and after 3 months for the clinical parameters like (Probing Pocket Depth (PPD), Clinical Attachment Level (CAL), Bleeding on Probing (BOP). In this study comparative analysis was carried out in which Diode Laser (800 - $980 \mathrm{~nm}$ ) was used as accessory to scaling and root planning (SRP) and conventional SRP for chronic periodontitis treatment. 40 patients were selected who had probing pocket depth of $\geq 5 \mathrm{~mm}$. In this study we designed our groups in such a way that each treatment group belonged to a separate quadrant of the mouth, wherein one group was allotted to SRP group and the other was allotted to SRP + Diode Laser group. For treating periodontitis, a nonsurgical approach i.e., diode Laser can be used as an adjunct to scaling and root Planing (SRP) which is a conventional method for improvement of CAL and also reduction of PPD.

The purpose of this study was to confirm whether the use of diode laser as adjunct to SRP improved the results of conventional mechanotherapy or conventional SRP alone in the treatment of chronic periodontitis.

\section{METHODS}

In this study we designed our groups so that there was total 160 sites from 40 individuals ( 20 males and 20 females) with two deepest nonadjacent pockets $\geq 5 \mathrm{~mm}$ in two different quadrants with CAL (clinical attachment level), BOP (bleeding on probing), PPD (pocket probing depth) $\geq 5 \mathrm{~mm}$. The present study was conducted in the Department of Periodontology, Faculty of Dental Sciences, from January 2014 to October 2015, SGT University after approval from ethical committee of the university. Inclusion criteria were probing depth of $\geq 5 \mathrm{~mm}$ in two non -adjacent teeth of two different quadrants and patients unwilling to undergo surgical treatment. Exclusion criteria included patients who underwent any periodontal treatment within past 12 months, antibiotics used within past 6 months, any sign of apical pathology, use of antiinflammatory drugs within the past 3 months, pregnancy, smoking and use of hormone contraceptives. All participating subjects had to sign informed consent form for their willingness to be involved in the study which was approved by ethical committee of the University.

\section{Pre-Treatment Protocol}

Study models were prepared for each subject, orthopantomogram and photographs were taken for record purposes. Resin stents were made for all pre and postoperative clinical reference measurement. Pre-operative photograph with stent (a) control site, (b) test site (Figure 1(a, b)).

\section{Clinical Parameters}

At baseline, post 1 month and post 3 months all clinical parameters such as Probing Pocket Depth (PPD), bleeding on Probing (BOP), Clinical Attachment Level (CAL) were 
measured and recorded. By inserting periodontal probe in the pocket the presence of bleeding was determined after 30 seconds. Periodontal probe UNC - 15 was used for the measurement of the PPD, ${ }^{11} \mathrm{CAL}^{11}$

\section{Treatment Procedure}

In every patient, the two non-adjacent deepest pockets $\geq 5 \mathrm{~mm}$ in two different quadrants were selected using a UNC - 15 periodontal probe, thus making a total of four sites in each selected patient. After clinical examination, the selected sites were randomly assigned as scaling and root planing alone (control) and Diode Laser as an accessory to scaling and root planning (SRP + DL) (test). Both sites were treated at the same visit. All clinical examinations which included PPD, BOP, CAL were measured at baseline and were repeated at 1 month and after 3 months (Figure 2, 3).

\section{SRP Procedure}

At test as well as control sites SRP was carried out under infiltration local anaesthesia (xylocaine $2 \%$ with adrenaline) using hand instruments and sonic devices in a single visit.

\section{Laser Procedure}

In the test site SRP was followed by laser irradiation during the same visit. Diode Laser (Sirona Dental SystemTM, Germany) was used for lasing the sites with a wavelength of $800-980$ $\mathrm{nm}$. The fiber optic laser tip of $320 \mu \mathrm{m}$ diameter was used at a power of $2.5 \mathrm{~W}$. The light guide was applied into the previously selected test sites by moving laser from apical to coronal direction, in a sweeping fashion which was parallel to the root surface. Lasing period was confined to the respective pocket depth such as $5 \mathrm{~mm}$ deep pockets were lased for 5 seconds, 6 $\mathrm{mm}$ deep pockets were lased for 6 seconds, $7 \mathrm{~mm}$ deep pockets were lased for 7 seconds and so on. ${ }^{12}$

\section{Statistical Analysis}

Formula for determining sample size $\mathrm{N}=4 \mathrm{PQ} / \mathrm{d}^{2}$

Where, $\mathrm{N}=$ sample size, $\mathrm{P}=$ Prevalence (from previous studies), $\mathrm{Q}=100-\mathrm{P}, \mathrm{d}=$ allowable error ( $5-20 \%$ of $\mathrm{P}$ ), $\mathrm{N}=4$ x $0.5(1-0.5) /(0.08)^{2}, \mathrm{~N}=156$

Probing depth and clinical attachment level values were normally distributed and analysed using the unpaired and paired $t$ test. When the difference in $P$ value was $<0.001$ then it was considered as statistically significant.

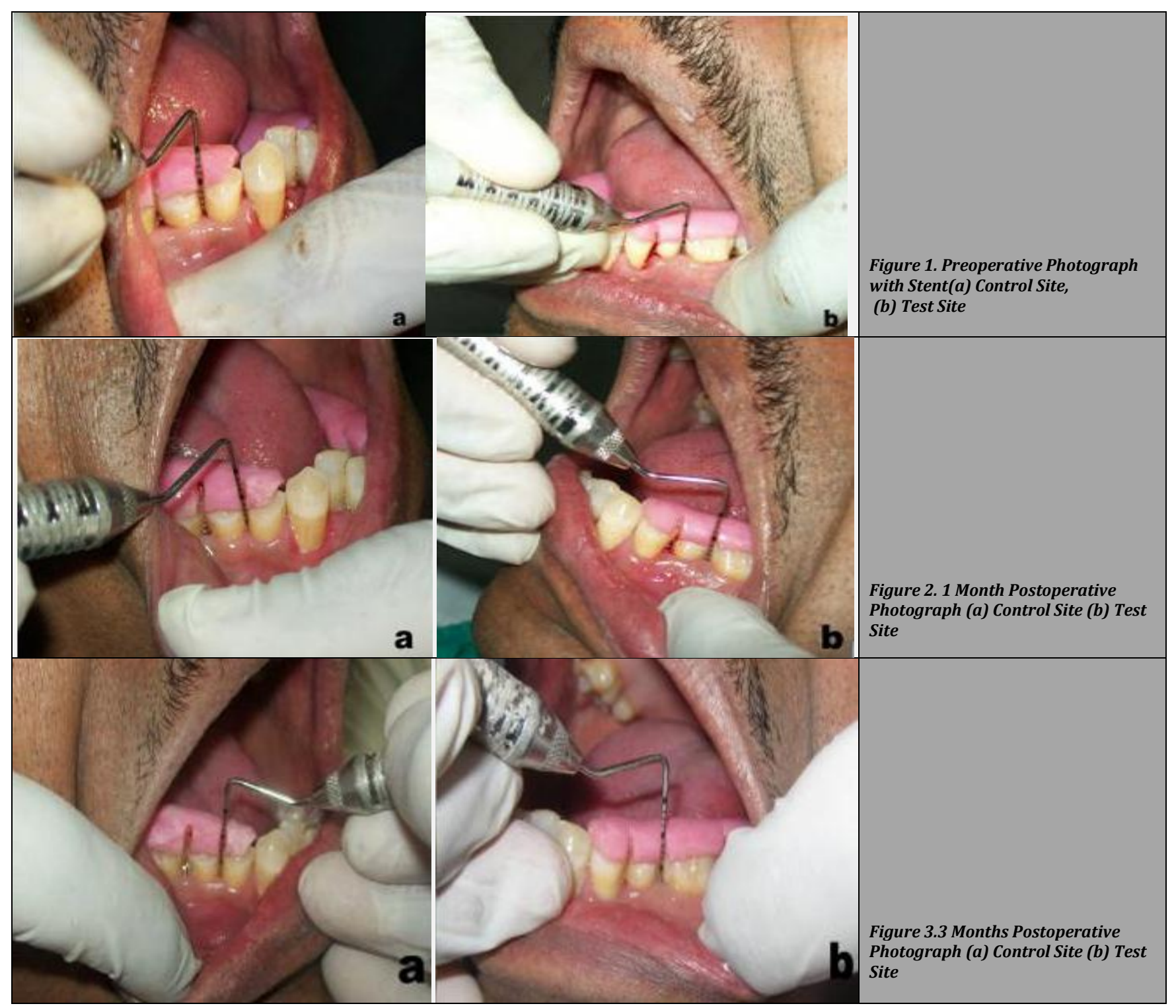




\begin{tabular}{|c|c|c|c|c|c|c|c|c|}
\hline Sex & & Time Intervals & $\mathbf{N}$ & Mean & Std. Deviation & Mean & Std. Deviation & P - Value \\
\hline \multirow{5}{*}{ Male } & \multirow{3}{*}{ SRP Group } & Baseline - 1month & 20 & 6.63 & 0.76 & 5.13 & 0.86 & $<0.001$ \\
\hline & & Baseline - 3months & 20 & 6.63 & 0.76 & 4.25 & 0.75 & $<0.001$ \\
\hline & & 1month - 3months & 20 & 5.13 & 0.86 & 4.25 & 0.75 & $<0.001$ \\
\hline & \multirow{2}{*}{ SRP + Laser Group } & Baseline - 1month & 20 & 7.20 & 0.86 & 5.10 & 0.84 & $<0.001$ \\
\hline & & 1month - 3months & 20 & 5.10 & 0.84 & 3.88 & 0.76 & $<0.001$ \\
\hline \multirow{5}{*}{ Female } & \multirow{3}{*}{ SRP Group } & Baseline - 1month & 20 & 6.65 & 0.73 & 5.03 & 0.70 & $<0.001$ \\
\hline & & Baseline - 3months & 20 & 6.65 & 0.73 & 4.10 & 0.70 & $<0.001$ \\
\hline & & 1month - 3months & 20 & 5.03 & 0.70 & 4.10 & 0.70 & $<0.001$ \\
\hline & \multirow{2}{*}{ SRP + Laser Group } & Baseline - 1month & 20 & 7.05 & 0.93 & 4.98 & 0.79 & $<0.001$ \\
\hline & & 1month - 3months & 20 & 4.98 & 0.79 & 3.73 & 0.70 & $<0.001$ \\
\hline \multicolumn{9}{|c|}{$\begin{array}{c}\text { Table 1. Intragroup Comparison of Mean PPD Values of Both Males and Females in SRP and SRP } \\
+ \text { Laser Group at Baseline, after } 1 \text { Month and after } 3 \text { Months }\end{array}$} \\
\hline
\end{tabular}

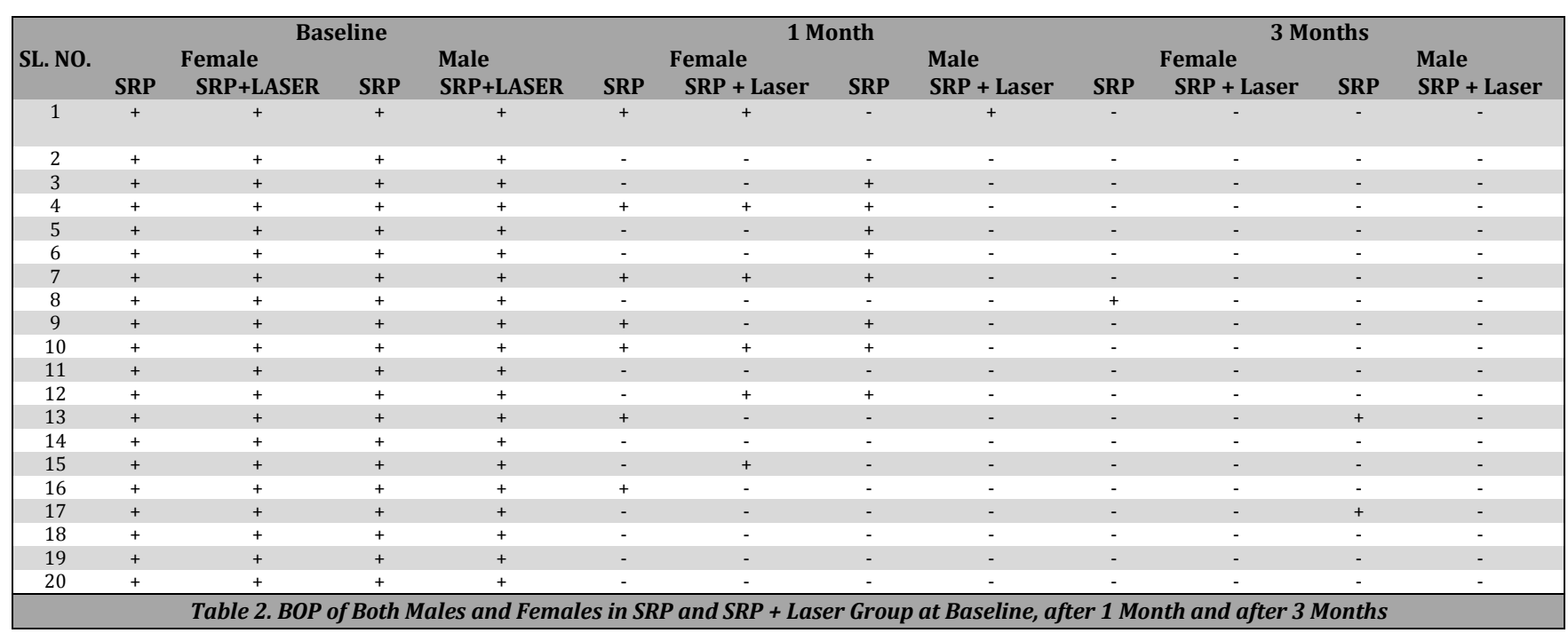

\begin{tabular}{|c|c|c|c|c|c|c|c|c|}
\hline Sex & & Time Intervals & $\mathbf{N}$ & Mean & Std. Deviation & Mean & Std. Deviation & P - Value \\
\hline \multirow{5}{*}{ Male } & \multirow{3}{*}{ SRP Group } & Baseline - 1month & 20 & 4.98 & 0.68 & 3.98 & 0.68 & $<0.0001$ \\
\hline & & Baseline - 3months & 20 & 4.98 & 0.68 & 3.08 & 0.47 & $<0.0001$ \\
\hline & & 1month - 3 months & 20 & 3.98 & 0.68 & 3.08 & 0.47 & $<0.0001$ \\
\hline & \multirow{2}{*}{ SRP + laser Group } & Baseline - 1month & 20 & 5.33 & 0.52 & 4.33 & 0.52 & $<0.0001$ \\
\hline & & 1month - 3months & 20 & 4.33 & 0.52 & 3.35 & 0.46 & $<0.0001$ \\
\hline \multirow{6}{*}{ Female } & \multirow{3}{*}{ SRP Group } & Baseline - 1month & 20 & 4.98 & 0.62 & 3.98 & 0.62 & $<0.0001$ \\
\hline & & Baseline - 3months & 20 & 4.98 & 0.62 & 3.05 & 0.43 & $<0.0001$ \\
\hline & & 1month - 3 months & 20 & 3.98 & 0.62 & 3.05 & 0.43 & $<0.0001$ \\
\hline & \multirow{3}{*}{ SRP + laser Group } & Baseline - 1month & 20 & 5.15 & 0.73 & 4.15 & 0.73 & $<0.0001$ \\
\hline & & Baseline - 3months & 20 & 5.15 & 0.73 & 3.23 & 0.55 & $<0.0001$ \\
\hline & & 1month - 3months & 20 & 4.15 & 0.73 & 3.23 & 0.55 & $<0.0001$ \\
\hline \multicolumn{9}{|c|}{$\begin{array}{l}\text { Table 3. Intra Group Comparison of Mean CAL Values of Both Males and Females in SRP and SRP } \\
+ \text { + Laser Group at Baseline, Post } 1 \text { Month and Post } 3 \text { Months }\end{array}$} \\
\hline
\end{tabular}

\section{RESULTS}

Table 1 shows that there was significant difference in mean PPD values of males and females in SRP group and SRP + Laser group between baseline and post 1 month $(\mathrm{P}<0.001)$, between baseline and post 3 months $(\mathrm{P}<0.001)$ and between 1 and 3 months $(\mathrm{P}<0.001)$. Table 2 shows that Bleeding on probing in females of SRP group was decreased in $65 \%$ cases at 1 month and decreased in $95 \%$ cases after 3 months, while in SRP + Laser group was decreased in $70 \%$ cases at 1 month and there was $100 \%$ decrease in bleeding on probing after 3 months. Similarly, bleeding on probing in males of SRP group was decreased in $60 \%$ cases at 1 month and decreased in 90 $\%$ of cases after 3 months, while in SRP + Laser group was decreased in $95 \%$ cases at 1 month and there was $100 \%$ decrease in bleeding on probing after 3 months.

\section{DISCUSSION}

Periodontal disease is a chronic infectious disease of the periodontium which affects soft \& mineralized tissue surrounding the teeth. Progression of the periodontal disease is associated with biofilm formation and also with the colonization of the sub gingival bacteria which aggravates degeneration of the collagen fibers which supports the tooth to the gingival and the alveolar bone, leading to alveolar bone resorption and chronic inflammation of soft tissues. ${ }^{13}$

Bacterial plaque (microbial biofilm), which is the primary aetiology of gingivitis and periodontitis is eliminated by the anti-infective therapy which comprises of both chemotherapeutic and mechanical approaches. The basis of the periodontal therapy is the eradication of local irritating factors and mechanical debridement of the dental biofilm. 
During the initial phase of periodontal therapy there is debridement of biofilm and also the eradication of local factors. Plaque and calculus and other plaque retentive local factors are effectively removed by the mechanical therapy which comprises of power driven or hand instruments for the debridement of the root surface. The term 'mechanical therapy' refers to both supra - gingival and sub - gingival scaling and root planning (SRP). ${ }^{14}$

For the sustainment of the gingival health patients are required to maintain a superior level of plaque control but most of the patients are unable to attain that level and hence other form of chemotherapy is needed. Now a days, chemotherapeutic agents are being used as accessories to nonsurgical anti-infective regimen because many patients are not trained in effective plaque removal. For the treatment of soft tissues, diode laser has been used but it does not remove calculus from the root surface, that's why diode laser is used as an accessory to SRP due to its detoxification \& bactericidal effect. 12

The selection criteria of present study was in accordance with the studies of Tomasi et al. ${ }^{15}$ Lopes et al. ${ }^{16}$ and Saglam et al. ${ }^{17}$ Un-cooperative patients, patients who had undergone periodontal treatment within the last 12 months, patients who had used anti-inflammatory drugs within last 3 months, patients on hormonal contraceptives, pregnant patients and also those patients with habit of tobacco chewing, smoking or any other habits that might influence the disease or the treatment were excluded from the study. ${ }^{16}$

The study had a split mouth design, in which 160 sites from 40 individuals (20 males and 20 females) 80 sites (40 sites in males and 40 sites in females) were assigned as control group in which SRP was performed and the other 80 sites ( 40 sites in males and 40 sites in females) were assigned as test group in which SRP + Laser application were performed.16,18 The selection of the sites for SRP and SRP + Laser application were done randomly. Ribeiro et al. ${ }^{19}$ also selected the sites randomly as control site and test site. The clinical assessment was done by measuring pocket probing depth (PPD), ${ }^{11}$ clinical attachment level (CAL) ${ }^{11}$ and bleeding on probing (BOP). ${ }^{16}$

All the parameters were recorded by single examiner to eliminate inter examiner variability. Clinical parameters were recorded for all the patients at baseline, post 1 month, and post 3 months' interval. 18,20

In present study each tooth was subdivided into four sides i.e., buccal, lingual / palatal, distal and mesial. Diode laser (800 - $980 \mathrm{~nm}$ ) was used with $2.5 \mathrm{~W}$ power in continuous mode. Kreisler et al. ${ }^{20}$ and Borrajo et al..$^{21}$ used diode laser with same specifications in their studies.

The diode laser was applied into the periodontal pockets by moving it from apical to coronal direction, in a sweeping fashion parallel to the root surface. Lasing period was confined to the respective pocket depth such as $5 \mathrm{~mm}$ deep pockets were lased for 5 seconds, $6 \mathrm{~mm}$ deep pockets were lased for 6 seconds, $7 \mathrm{~mm}$ deep pockets were lased for 7 seconds and so on. Moritz et al. ${ }^{12}$ used same lasing criteria.

\section{Probing Pocket Depth (PPD)}

Moritz et al. ${ }^{12}$ used diode laser in their studies, they found that patients treated with laser as adjuvant to scaling and root planing showed more statistically significant results as compared to conventional mechanical therapy. In our study, a significant curtailment of PPD was observed in both groups after treatment. Kriesler et al. ${ }^{20}$ recommended that more curtailment of PPD may be related to the de-epithelization of the pockets which gives rise to increased connective tissue attachment. Our result implies that there is no substantial inflammatory difference as compared to the mechanical therapy alone while resulting in an enhanced clinical healing.

\section{Bleeding on Probing (BOP)}

Periodontal destruction is a continuous pattern due to the repeated BOP in one site. Eradication of bleeding on probing and improvement of the clinical sign of inflammation can be expected due to the phase I therapy.

In fact, we observed that the there is more curtailment of BOP in SRP + Laser group as compared to SRP group alone. Borrajo et al. ${ }^{21}$ have studied diode laser is used as an adjunctive to scaling and root planing in a duration of 6 weeks. The results were in accordance with the present study in which BOP reduced in both groups but this reduction was more in SRP + DL group as compared to the scaling and root planing group.

In contrast to the present study, Kreisler ${ }^{20}$ and Kelbauskiene ${ }^{22}$ didn't show any significant differences between SRP group and SRP + Laser group.

\section{Clinical Attachment Level (CAL)}

Borrajo et al. ${ }^{21}$ and Caruso et al. ${ }^{18}$ used diode laser in their studies, they demonstrated that patients treated with laser as adjunctive therapy to SRP showed more statistically significant results in comparison to those treated with conventional mechanical therapy. In present study, a significant gain in CAL was shown in both groups after completion of the treatment was proved.

\section{CONCLUSIONS}

Although the results may justify the protocol, comparing SRP group with diode laser as adjunct to SRP group in chronic periodontitis treatment, has significantly improved the clinical parameters (PPD, BOP, CAL). When SRP and SRP + Diode laser groups were compared they showed statistically nonsignificant results but individually both groups showed statistically significant results. A cost - benefit analysis should carefully be considered in light of high investment required for this treatment which may not justify this limited added benefit. A newer approach for the treatment of chronic periodontitis with laser has been used as an accessory treatment along with the SRP or Lacing replacing SRP, but these can be performed with caution until there are several randomized controlled trials with sufficient statistical power.

\section{Limitation}

These results must also be considered with utmost care in light of limitations of this clinical trial, short duration of the study, 
limited sample size, and the differences between the tested treatment protocols, which may complicate a direct comparative analysis.

Data sharing statement provided by the authors is available with the full text of this article at jemds.com.

Financial or other competing interests: None.

Disclosure forms provided by the authors are available with the full text of this article at jemds.com.

\section{REFERENCES}

[1] Brito F, Almeida S, Figueredo CMS, et al. Extent and severity of chronic periodontitis in chronic kidney disease patients. J Periodont Res 2012;47(4):426-30.

[2] Fleischer HC, Mellonig JT, Brayer WK, et al. Scaling and root planning efficacy in multirooted teeth. J Periodontol 1989;60(7):402-9.

[3] Ivic-Kardum M, Jurak I, Gall-Troselj K, et al. The effect of scaling and root planing on the clinical and microbiological parameters of periodontal diseases. Acta Stomatol Croat 2001;35(1):39-42.

[4] Adriaens PA, Adriaens LM. Effects of nonsurgical periodontal therapy on hard and soft tissues. Periodontol 2000 2004;36(1):121-45.

[5] Adriaens PA, Edwards CA, DeBoever JA, et al. Ultrastructural observations on bacterial invasion in cementum and radicular dentin of periodontally diseased human teeth. J Periodontol 1988;59(8):493-503.

[6] Sbordone L, Ramaglia L, Gulleta E, et al. Recolonization of the subgingival microflora after scaling and root planning in human periodontitis. J Periodontol 1990;61(9):579-84.

[7] Sgolastra F, Severino M, Gatto R, et al. Effectiveness of diode laser as adjunctive therapy to scaling root planning in the treatment of chronic periodontitis: a meta-analysis. Lasers Med Sci 2013;28(5):1393-402.

[8] Darveau RP, Tanner A, Page RC. The microbial challenge in periodontitis. Periodontol 2000 1997;14(1):12-32.

[9] Haveman KW, Wilson JH. Laser in periodontics: an overview. Clinical Update 2011;33(1):1-2.

[10] Lee DH. Application of laser in periodontics: a new approach in periodontal treatment. Dental Bulletin 2007;12(10):23-5.
[11] Isidor F, Karring T, Attstrom R. Reproducibility of pocket depth and attachment level measurements when using a flexible splint. J Clin Periodontol 1984;11(10):662-8.

[12] Moritz A, Schoop U, Goharkhay K, et al. Treatment of periodontal pockets with a diode laser. Lasers Surg Med 1998;22(5):302-11.

[13] Krayer JW, Leite RS, Kirkwood KL. Non-surgical chemotherapeutic treatment strategies for the management of periodontal diseases. Dent Clin North Am 2010;54(1):13-33.

[14] Drisko CH. Nonsurgical periodontal therapy. Periodontol 2000 2001;25(1):77-88.

[15] Tomasi C, Schander K, Dahlen G, et al. Short-term clinical and microbiologic effects of pocket debridement with an Er: YAG laser during periodontal maintenance. J Periodontol 2006;77(1):111-8.

[16] Lopes BMV, Theodoro LH, Melo RF, et al. Clinical and microbiologic follow-up evaluations after non-surgical periodontal treatment with Erbium: YAG laser and scaling and root planing. J Periodontol 2010;81(5):682-91.

[17] Saglam M, Kantarci A, Dundar N, et al. Clinical and biochemical effects of diode laser as an adjunct to non surgical treatment of chronic periodontitis: a randomized, controlled clinical trial. Lasers Med Sci 2014;29(1):37-46.

[18] Caruso U, Nastri L, Piccolomini R, et al. Use of diode laser $980 \mathrm{~nm}$ as adjunctive therapy in the treatment of chronic periodontitis. A randomized controlled clinical trial. New Microbiol 2008;31(4):513-8.

[19] Ribeiro IW, Sbrana MC, Esper LA, et al. Evaluation of the effect of the GaAlAs laser on subgingival scaling and root planing. Photomed Laser Surg 2008;26(4):387-91.

[20] Kreisler M, Haj HA, D'Hoedt B. Clinical efficacy of semiconductor laser application as an adjunct to conventional scaling and root planing. Lasers Surg Med 2005;37(5):350-5.

[21] Borrajo JLL, Varela G, Castro GL, et al. Diode laser as adjunct to scaling and root planing. Photomed Laser Surg 2004;22(6):509-12.

[22] Kelbauskiene S, Maciulskiene V. A pilot study of Er, Cr: YSGG laser therapy used as an adjunct to scaling and root planing in patients with early and moderate periodontitis. Stomatologija 2007;9(1):21-6. 